\title{
A Note on the Statistical Approximation Properties of the Modified Discrete Operators
}

\author{
Reyhan Canatan \\ Ankara University, Department of Mathematics, Ankara, Turkey \\ Email: reyhan.canatan@gmail.com
}

Received April 22, 2012; revised May 27, 2012; accepted June 12, 2012

\begin{abstract}
In this present paper, firstly, the modified positive operators and its discrete operators are constructed. Then, we investigate the statistical approximation properties and rates of convergence by using modulus of continuity of these positive linear operators. Finally, we obtain the rate of statistical convergence of truncated operators.
\end{abstract}

Keywords: Sequence of Positive Linear Operators; Bohman-Korovkin Theorem; Statistical Approximation; Modulus of Continuity; Rate of Convergence

\section{Introduction}

First of all, let us recall the concept of statistical convergence. The natural density (or density) of the set $K \subset I N$ is denoted as $\delta(K)$.

For $\delta(K):=\lim _{n} \frac{1}{n}\{$ the number $k \leq n: k \in K\}$

whenever the limit exists (see e.g. [1]) if for every

$$
\varepsilon>0, \delta\left\{k \in N:\left|x_{k}-L\right| \geq \varepsilon \mid\right\}=0
$$

then we say that a sequence $x=\left(x_{k}\right)$ is said to be statistcally convergent to a number of $L$ (see Fast in [2]).

The concept of statistical convergence is very important in approximation theory because although any sequence which is convergent in ordinary sense is statistically convergent, but contrary can not be true all the time. For instance;

If we choose $\left(x_{k}\right)$ as

$$
x_{k}=\left\{\begin{array}{ll}
L_{1} & n=m^{2} \\
L_{2} & n \neq m^{2}
\end{array} \quad(m=1,2,3, \cdots)\right\}
$$

then we can easily say that it is statistically convergent to $L_{2}$ but not convergent in ordinary sense when $L_{1} \neq L_{2}$. Recently, linear positive operators and their Korovkin type statistical approximation properties have been investigated by many authors. It is well-known that lots of operators were defined with infinite series. Details can be found in [3]. For example, $n$-th Favard-Mirakjan-Szász operator was defined by

$$
\left(S_{n} f\right)(x):=\sum_{k=0}^{\infty} f\left(\frac{k}{n}\right) s_{n, k}(x), s_{n, k}(x)=\frac{(n x)^{k}}{k !} e^{-n x}
$$

for every $f$ belonging to Banach lattice $E_{2}, x \in[0, \infty)$ and $n \in I N$, where

$$
E_{2}:=\left\{f \in C([0, \infty)): \lim _{x \rightarrow \infty} \frac{f(x)}{1+x^{2}} \text { is finite }\right\}
$$

is endowed with the norm $\|f\|_{E_{2}}:=\sup _{x \geq 0} \frac{|f(x)|}{1+x^{2}}$.

In [4], Doğru investigate the weighted approximation properties of general positive linear operators on infinite intervals. Later, in 2002, weighted approximation properties of Szász-type operators are investigated by same author in [5]. In this note, we investigate the statistical approximation properties considering only the partial sums of the operators. In [6], J. Grof studied on the operator

$$
\left(S_{n, N} f\right)(x):=\sum_{k=0}^{N} f\left(\frac{k}{n}\right) S_{n, k}(x)
$$

where $s_{n, k}(x)=\frac{(n x)^{k}}{k !} e^{-n x}$ and he verified that if $(N(n))_{n \geq 1}$ is a sequence of positive integers such that $\lim _{n \rightarrow \infty} \frac{N(n)}{n}=\infty$ then $\lim _{n \rightarrow \infty}\left(S_{n, N} f\right)(x)=f(x)$ for all $x \geq 0$ and $f \in C[0, \infty)$. Here, $f$ satisfies the inequality

$$
|f(t)| \leq A e^{m t}\left(A \in R^{+}, m \in N\right) .
$$

In 1984, Heintz-Gerd Lehnhoff [7] studied the following Modified Szász operators

$$
\left(S_{n, \delta} f\right)(x):=\sum_{k=0}^{[n(x+\delta)]} f\left(\frac{k}{n}\right) s_{n, k}(x), x \geq 0
$$


where $s_{n, k}(x)=\frac{(n x)^{k}}{k !} e^{-n x}, f \in C[0, \infty)$.

Grof and Lehnhoff obtained the conditions which ensure the convergence of the operators $\left(S_{n, \delta} f\right)$ to $f$.

Notice that the notation $[\gamma]$ shows the largest integer and it is not exceeding the number $\gamma$.

The main aim of this paper is to investigate the statistical approximation properties of the operators which constructed and examined the ordinary approximation properties by Agratini in [8].

\section{Statistical Approximation Properties}

Let us recall the operators which were defined by Agratini in [8].

Throughout the paper $I N_{0}=\{0\} \cup I N, K$ indicates a compact subinterval of $I R^{+}$and $e_{j}$, the $j$-th monomial, $e_{j}(t)=t^{j}$.

Let us assume that the following cases for each $n \in N$,

1) For every $k \in I N_{0}$, a sequence of $\gamma_{k}$ exists such that

$$
x_{n, k}=O\left(n^{-\gamma_{k}}\right)(n \rightarrow \infty)
$$

a net on $I R^{+}, \Delta_{n}=\left(x_{n, k}\right)_{k \geq 0}$ is fixed.

2) There is a sequence $\left(\phi_{n, k}\right)_{k \geq 0}$ such that

$\phi_{n, k} \in C^{\prime}\left(I R^{+}\right)$. Where, $C^{\prime}\left(I R^{+}\right)$is the space of all realvalued functions continuously differentiable in $I R^{+}$.

For this sequence $\left(\phi_{n, k}\right)_{k \geq 0}$ the following conditions

$$
\phi_{n, k} \geq 0, k \in I N_{0}, \sum_{k=0}^{\infty} \phi_{n, k}=e_{0}, \sum_{k=0}^{\infty} x_{n, k} \phi_{n, k}=e_{1}
$$

hold.

3) A positive function $\psi \in I R^{I N \times I R^{+}}, \psi(n,.) \in C\left(I R^{+}\right)$, exists with the property,

$$
\psi(n, x) \phi_{n, k}^{\prime}(x)=\left(x_{n, k}-x\right) \phi_{n, k}(x), k \in I N_{0}, x \geq 0 .
$$

By using these requirements the operators were defined as

$$
L_{n,}(f ; x):=\sum_{k=0}^{\infty} \phi_{n, k}(x) f\left(x_{n, k}\right), x \geq 0, f \in F
$$

where $F$ stands for the domain of $L_{n}$ containing the set of all continuous functions on $I R^{+}$for which the series in (5) is convergent.

We note that, with specific choosing these operators turn into the operators mentioned in [1].

Lemma A. [8] Let $L_{n}, n \in I N$, be defined by (5) and $\varphi_{n, r}$ be the r-th central moment of $L_{n}$. For every $x \in I R^{+}$, we have the following identities,

$$
\varphi_{n, 0}(x)=1, \varphi_{n, 1}(x)=0,
$$

$$
\begin{gathered}
\varphi_{n, r+1}(x)=\psi(n, x)\left(\varphi_{n, r}^{\prime}(x)+r \varphi_{n, r-1}(x)\right), r \in I N, \\
\varphi_{n, 2}(x)=\psi(n, x) .
\end{gathered}
$$

A Korovkin type statistical approximation theorem for any sequence of positive linear operators was proved by Gadjiev and Orhan in [9]. First, let us recall this theorem. Where $C_{M}[a, b]$ denotes all functions $f$ that are continuous in $[\mathrm{a}, \mathrm{b}]$ and bounded all positive axis.

Theorem A. [9] If the sequence of positive linear operators $A_{n}: C_{M}[a, b] \rightarrow C[a, b]$ satisfies the conditions

$$
s t-\lim _{n}\left\|A_{n} e_{j}-e_{j}\right\|_{C[a, b]}=0, e_{j}(t)=t^{j}, j=0,1,2
$$

then for any function $f \in C_{M}[a, b]$ we have,

$$
s t-\lim _{n}\left\|A_{n} f-f\right\|_{C[a, b]}=0 .
$$

Now, we can give the following theorem which includes the satatistical convergence of the operators in (5).

Theorem 1. Let $L_{n}$, be the operators defined in (5). If st $-\lim \psi(n, x)=0$, uniformly on $K$ then for every $f \in F^{n}$ we have,

$$
s t-\lim _{n}\left\|L_{n}(f ; .)-f\right\|_{C(K)}=0 .
$$

Proof. Because of (3) we can easily say that

$$
s t-\lim _{n}\left\|L_{n}\left(e_{0} ; .\right)-e_{0}\right\|_{C(K)}=0,
$$

and

$$
s t-\lim _{n}\left\|L_{n}\left(e_{1} ; .\right)-e_{1}\right\|_{C(K)}=0 .
$$

We know from (8) that $\psi(n, x)=\varphi_{n, 2}(x)=L_{n}\left((t-x)^{2} ; x\right)$. By using the linearity of the operator

$$
\psi(n, x)=L_{n}\left(t^{2} ; x\right)-2 x L_{n}(t ; x)-x^{2} L_{n}(1 ; x) .
$$

From (3)

$$
\psi(n, x)=L_{n}\left(e_{2} ; x\right)-2 x x-x^{2}=L_{n}\left(e_{2} ; x\right)-x^{2} .
$$

Hence,

$$
\left\|L_{n}\left(e_{2} ;\right)-e_{2}\right\|_{C(K)}=\|\psi(n, x)\|_{C(K)} .
$$

In view of $s t-\lim _{n} \psi(n, x)=0$ we have

$$
s t-\lim _{n}\left\|L_{n}\left(e_{2} ; .\right)-e_{2}\right\|_{C(K)}=0 .
$$

Now, we are able to say in the light of Theorem A that $s t-\lim _{n}\left\|L_{n}(f ; .)-f\right\|_{C(K)}=0$ which ends the proof.

By using modulus of continuity, we mention about the rate of statistical convergence of these operators. First, let us remember the definition of modulus of continuity. Let $f \in C(K), \omega(f ; \delta)$ the modulus of continuity of $f$, is defined as 


$$
\omega(f ; \delta)=\sup _{\substack{x, t \in K \\|t-x|<\delta}}|f(t)-f(x)| .
$$

Let $L_{n}$ be defined by (5), for every $f \in C_{B}\left(I R^{+}\right)$, $x \geq 0$ and $\delta>0$. We know from Theorem 1 in [8] that

$$
\left|L_{n}(f ; x)-f(x)\right| \leq\left(1+\delta^{-1} \sqrt{\psi(n, x)}\right) \omega(f ; \delta)
$$

If we take norm on $K$ and choose $\delta=\delta_{n}=\left[\psi(n, x)^{1 / 2}\right]$, we get $\left\|L_{n}(f ; .)-f\right\|_{C(K)} \leq 2 \omega\left(f ; \delta_{n}\right)$. Due to $s t-\lim _{n} \psi(n, x)=0$, we have the rates of statistical convergence of the operators in (5).

\section{Modified Discrete Operators}

In this section, we recall the modified discrete operators which were defined by Agratini in [8] and investigate the statistical approximation properties of these operators. If we specialize the net $\Delta_{n}$ and function $\psi$ respectively,

$$
\begin{aligned}
& x_{n, k}=\frac{k}{a_{n}} \leq k, s t-\lim _{n}\left(a_{n}\right)^{-1} \\
& \psi_{n, x}=\sum_{i=1}^{l} \frac{\psi_{i}(x)}{a_{n}^{i}}, x \geq 0
\end{aligned}
$$

under these assumptions, the requirement of Theorem 1 is fulfilled. Starting from (5) under the additional assumptions (14) Agratini defined,

$$
\begin{aligned}
& L_{n, \delta}(f ; x):=\sum_{k=0}^{\left[a_{n}(x+\delta(n))\right]} \varphi_{n, k}(x) f\left(\frac{k}{a_{n}}\right), \\
& x \geq 0, f \in F
\end{aligned}
$$

where, $\delta=(\delta(n))_{n \geq 1}$ is a sequence of positive numbers. The study of these operators were developed in polynomial weighted spaces connected to the weights $\omega_{m}, m \in I N_{0}, \omega_{m}(x)=\left(1+x^{2 m}\right)^{-1}, x \geq 0$. For every $m \in$ $I N_{0}$ the spaces

$$
E_{m}:=\left\{f \in C\left(I R^{+}\right):\|f\|_{m}:=\sup _{x \geq 0} \omega_{m}(x)|f(x)|<\infty\right\}
$$

endowed with the norm $\|\cdot\|_{m}$.

Lemma B. [8] Let $L_{n}$, be defined by (5) and the assumptions (14) are fulfilled. If, $\psi_{i} \in C^{2 m-2}\left(I R^{+}\right)$, $i=1,2, \cdots l$ then the central moment of $2 m$-th order verifies

$$
\varphi_{n, 2 m} \leq \frac{C(m, K)}{a_{n}^{m}}, x \in K .
$$

Where $C(m, K)$ is a constant depending only on $m$ and the compact $K$.

Theorem 2. Let $L_{n, \delta}$, be defined by (15). If

$$
\begin{aligned}
& \psi_{i} \in C^{2 m-2}\left(I R^{+}\right), i=1,2, \cdots, l, \\
& s t-\lim _{n}\left[\sqrt{a_{n}} \delta(n)\right]^{-1}=0, \\
& s t-\lim _{n}\left\|L_{n, \delta}(f ; .)-f\right\|_{C(K)}=0,
\end{aligned}
$$

holds for every $f \in E_{m} \cap F$.

Proof. We use the following,

$$
\begin{aligned}
& t^{2 m} \leq 2^{2 m-1}\left(x^{2 m}+(t-x)^{2 m}\right), \\
& t \geq 0, x \geq 0, m \in I N
\end{aligned}
$$

and for $a_{f}, b_{f}$ which are the positive constants $|f| \leq a_{f}+b_{f} e_{2 m}$ hold true. Hence,

$$
\begin{aligned}
& |f(t)| \leq g_{m}(x)+2^{2 m-1} b_{f}(t-x)^{2 m}, \\
& g_{m}:=a_{f}+b_{f} 2^{2 m-1} e_{2 m} .
\end{aligned}
$$

From this inequality,

$$
\begin{aligned}
& \left|f\left(\frac{k}{a_{n}}\right)\right| \leq g_{m}(x)+2^{2 m-1} b_{f}\left(\frac{k}{a_{n}}-x\right)^{2 m}, \\
& k \in I N_{0}, x \geq 0 .
\end{aligned}
$$

If $k \geq\left[a_{n}(x+\delta(n))\right]+1$ then $\frac{k}{a_{n}} \geq x$. On the grounds of $x, \delta(n)$ and $a_{n}$ are positive we can write that

$$
\begin{aligned}
& \left\{k \in I N_{0}: k \geq\left[a_{n}(x+\delta(n))\right]+1\right\} \subset \\
& \left\{k \in I N_{0}:\left|\frac{k}{a_{n}}-a_{n}\right|>\delta(n)\right\}:=I_{n, x, \delta}
\end{aligned}
$$

The remaining term is $R_{n}:=L_{n}-L_{n, \delta}$ and taking into consideration both (18) and (19)

$$
\begin{aligned}
\left|R_{n}(f ; x)\right| \leq & \sum_{k \in I_{n, x, \delta}} \varphi_{n, k}(x)\left|f\left(\frac{k}{a_{n}}\right)\right| \\
\leq & \sum_{k \in I_{n, x, \delta}} \varphi_{n, k}(x) g_{m}(x)+2^{2 m-1} b_{f} \\
& \times \sum_{k \in I_{n, x, \delta}} \varphi_{n, k}(x)\left(\frac{k}{a_{n}}-x\right)^{2 m} \\
\leq & g_{m}(x) \delta^{-2 m}(n) \sum_{k=0}^{\infty} \varphi_{n, k}(x)\left(\frac{k}{a_{n}}-x\right)^{2 m} \\
& +2^{2 m-1} b_{f} \phi_{n, 2 m}(x) \\
= & g_{m}(x) \delta^{-2 m}(n) \phi_{n, 2 m}(x) \\
& +2^{2 m-1} b_{f} \phi_{n, 2 m}(x) .
\end{aligned}
$$

By using (16)

$$
\left|R_{n}(f ; x)\right| \leq\left(g_{m}(x) \delta^{-2 m}(n)+2^{2 m-1} b_{f}\right) \frac{C(m, K)}{a_{n}^{m}}
$$

If we take norm on $K$ we have the following. 


$$
\begin{aligned}
\left\|R_{n}(f ; x)\right\|_{C(K)} & \leq\left\|g_{m}\right\| C(m, K)\left[\sqrt{a_{n}} \delta(n)\right]^{-2 m} \\
& +2^{2 m-1} b_{f} C(m, K) a_{n}^{-m} .
\end{aligned}
$$

By considering the concept of statistical convergence let us define the sets,

$$
\begin{aligned}
& E:=\left\{k \leq n:\left\|R_{k}(f ; x)\right\| \geq \varepsilon\right\} \\
& E_{1}:=\left\{k \leq n:\left[\sqrt{a_{k}} \delta(k)\right]^{-2 m} \geq \frac{\varepsilon}{2\left\|g_{m}\right\| C(m, K)}\right\} \\
& E_{2}:=\left\{k \leq n: a_{k}^{-m} \geq \frac{\varepsilon}{2^{2 m} b_{f} C(m, K)}\right\} .
\end{aligned}
$$

It is obvious that $E \subset E_{1} \cup E_{2}$ and $\delta(E) \leq \delta\left(E_{1}\right)+\delta\left(E_{2}\right)$ because of $s t-\lim _{n}\left[\sqrt{a_{n}} \delta(n)\right]^{-1}=0$ and $s t-\lim _{n} a_{n}^{-1}=0$.

The proof is completed.

\section{REFERENCES}

[1] I. Niven, H. S. Zuckerman and H. Montgomery, "An Introduction to the Theory of Numbers," 5th Edition, Wiley, New York, 1991.
[2] H. Fast, "Sur La Convergence Statistique," Colloquium Mathematicum, Vol. 2, 1951, pp. 241-244.

[3] O. Doğru, "On Statistical Approximation Properties of Stancu Type Bivariate Generalization of q-Balazs-Szabados Operators," Proceedings of International Conference on Numerical Analysis and Approximation Theory, Cluj-Napoca, 5-8 July 2006, pp. 179-194.

[4] O. Doğru, "On Weighted Approximation of Continuous Functions by Linear Positive Operators on Infinite Intervals," Mathematica, Vol. 41, No. 1, 1999, pp. 39-46.

[5] O. Doğru, "Weighted Approximation Properties of Szásztype Operators," International Journal of Mathematics, Vol. 2, 2002, pp. 889-895.

[6] J. Grof, "Approximation durch Polynome mit Belegfunktionen," Acta Mathematica Hungarica, Vol. 35, No. 1-2, 1980, pp. 109-116. doi:10.1007/BF01896829

[7] H. G. Lehnhoff, “On a Modified Szász-Mirakjan Operator," Journal of Approximation Theory, Vol. 42, 1984, pp. 278-282. doi:10.1016/0021-9045(84)90045-5

[8] O. Agratini, "On the Convergence of a Truncated Class of Operators," Bulletin of the Institute of Mathematics Academia Sinica, Vol. 312, No. 3, 2003, pp. 213-223.

[9] A. D. Gadjiev and C. Orhan, "Some Approximation Theorems via Statistical Convergence," Rocky Mountain Journal of Mathematics, Vol. 32, No. 1, 2002, pp. 129-138. doi: $10.1216 / \mathrm{rmjm} / 1030539612$ 